\title{
Digenic mutational inheritance of the integrin alpha 7 and the myosin heavy chain 7B genes causes congenital myopathy with left ventricular non-compact cardiomyopathy
}

Teresa Esposito ${ }^{1 *}$, Simone Sampaolo ${ }^{2}$, Giuseppe Limongelli³, Antonio Varone ${ }^{4}$, Daniela Formicola1,2, Daria Diodato ${ }^{2}$, Olimpia Farina ${ }^{2}$, Filomena Napolitano ${ }^{1}$, Giuseppe Pacileo ${ }^{3}$, Fernando Gianfrancesco ${ }^{1}$ and Giuseppe Di lorio²

\begin{abstract}
Background: We report an Italian family in which the proband showed a severe phenotype characterized by the association of congenital fiber type disproportion (CFTD) with a left ventricular non-compaction cardiomyopathy (LVNC). This study was focused on the identification of the responsible gene/s.

Methods and results: Using the whole-exome sequencing approach, we identified the proband homozygous missense mutations in two genes, the myosin heavy chain 7B (MYH7B) and the integrin alpha 7 (ITGA7). Both genes are expressed in heart and muscle tissues, and both mutations were predicted to be deleterious and were not found in the healthy population.

The R890C mutation in the MYH7B gene segregated with the LVNC phenotype in the examined family. It was also found in one unrelated patient affected by LVNC, confirming a causative role in cardiomyopathy.

The E882K mutation in the ITGA7 gene, a key component of the basal lamina of muscle fibers, was found only in the proband, suggesting a role in CFTD.

Conclusions: This study identifies two novel disease genes. Mutation in MYH7B causes a classical LVNC phenotype, whereas mutation in ITGA7 causes CFTD. Both phenotypes represent alterations of skeletal and cardiac muscle maturation and are usually not severe. The severe phenotype of the proband is most likely due to a synergic effect of these two mutations.

This study provides new insights into the genetics underlying Mendelian traits and demonstrates a role for digenic inheritance in complex phenotypes.
\end{abstract}

Keywords: Left ventricular noncompact cardiomyopathy, Congenital type fiber disproportion, Integrin alpha 7 (ITGA7), Myosin heavy chain 7B (MYH7B), Whole exome sequencing

\footnotetext{
* Correspondence: teresa.esposito@igb.cnr.it

'Institute of Genetics and Biophysics "Adriano Buzzati-Traverso", National

Research Council of Italy, Naples, Italy

Full list of author information is available at the end of the article
} 


\section{Background}

Congenital fiber type disproportion (CFTD) is a form of congenital myopathy in which consistent type 1 fiber hypotrophy relative to type 2 fibers is the main histological abnormality [1]. The health impairments commonly encountered in CFTD are similar to those of many other congenital myopathies. Muscle weakness is usually relatively stable or slowly progressive during childhood and adolescence. In general, scoliosis and joint contractures (other than mild Achilles tendon contractures) are relatively uncommon. Mutations associated with CFTD have been found in the TPM3 (MIM\# 191030), ACTA1 (MIM\# 102610), SEPN1 (MIM\# 606210), RYR1 (MIM\# 180901), TPM2 (MIM\# 190990) and MYH7 (MIM\# 160760) genes [2-8]. Despite recent advances, no genetic cause has been found in at least $50 \%$ of CFTD patients.

Left ventricular non-compaction cardiomyopathy (LVNC) has been reported in patients with different types of neuromuscular disorders but has never been associated with CFTD. Within the last 2 decades, more than 200 cases of LVNC have been described [9]. The hallmark features of this cardiomyopathy include prominent trabeculations and deep endocardial recesses. Symptoms associated with LVNC are variable and can include arrhythmias, thromboembolic events and heart failure. At least 4 genes that cause very rare cardiomyopathy diseases have been found to be linked to non-syndromic LVNC in familial or sporadic cases. Thus far, the X-linked gene known as G4.5 or taffazin (TAZ) has been found to be associated with the largest number of LVNC cases [10]. Other genes found to be mutated in LVNC include alpha-dystrobrevin (DTNA), Cypher/ZASP and lamin A/C [11-13]. Cumulatively, these genes have been shown to be associated with only a small percentage of sporadic or familial cases. Over the past few years, mutations in genes encoding sarcomere proteins, the beta-myosin heavy chain (MYH7), alpha-cardiac actin (ACTC1), cardiac troponin T (TNNT2), cardiac myosinbinding protein C (MYBPC3), alpha-tropomyosin (TMP1) and cardiac troponin I (TNNI3) have been identified in a significant proportion of patients with LVNC [14-16].

We report an Italian family in which the proband showed a severe disease phenotype characterized by CFTD and LVNC. The clinical and instrumental analysis of the family members identified the LVNC phenotype in the mother, the sister and the first-degree cousin of the proband, suggesting that the LVNC cardiomyopathy segregated as a dominant mode of inheritance with high phenotypic heterogeneity and reduced penetrance. CFTD phenotype showed a recessive mode of inheritance.

To our knowledge, this is the first example of LVNC cardiac phenotype associated with CFTD. We provide a full description of this new phenotype and of the identification of the disease-causing genes by whole-exome sequencing [17].

\section{Patients and methods}

\section{Family and unrelated cohort}

A single, multigenerational Italian pedigree was involved in this investigation, as depicted in Figure 1. Phenotypic data were available for 10 individuals ( 3 males and 7 females) ranging in age from 4 to 50 . All individuals were of Caucasian ancestry. DNA was available for 6 individuals (IV-10, IV-13, IV-14, V-1, V-4, V-5). We extended the clinical and instrumental (EMG, ECG, Echocardiography, muscle biopsy) evaluation to the whole family. We identified a mild LVNC phenotype (according to the Jenni criteria) in the mother (IV-14), sister (V-5) and cousin (V-1) of the proband, with hypertabeculations of the mid inferolateral wall and of the ape and without neuromuscular disorders [18]. During the growth, there was a reduction of clinical signs in patients $\mathrm{V}-1$ and $\mathrm{V}-5$. No data have been collected for individual IV-9, who died at the age of 35 of unknown causes. The father (IV-13) of the proband was asymptomatic.

An unrelated cohort (UC) of 24 patients (including LVNC14-BG), all fulfilling the criteria for LVNC, were also analyzed in this study.

Informed consent was obtained from each patient. In the case of minors, parental consent was obtained. The study protocol conforms to the ethical guidelines of the 1975 Declaration of Helsinki, as reflected in the a priori approval by the institution's human research committee.

\section{Whole-exome sequencing strategy}

For all participants over 18 years of age, DNA was extracted from peripheral blood specimens using a standard saltingout procedure. Genomic DNA samples from two affected females in the pedigree (the proband V-4, affected by CFTD and LVNC, and her cousin V-1, affected by LVNC) were captured with the NimbleGen SeqCap EZ Exome ${ }^{\mathrm{rm}}$ capture kits (Roche, Indianapolis, IN, USA)) and sequenced with one lane per sample on an Illumina GAIIx (Illumina, San Diego, CA, USA) with 90-bp pairedend reads.

Sequences were aligned to the human reference genome sequence (GRCh37/hg19) with the MAQ7 and NextGENe software v2.00 with sequence condensation by consolidation (SoftGenetics, State College, PA, USA). This approach resulted in more than $40 \times$ of target exome coverage.

Single nucleotide variants (SNVs) were called with MAQ and NextGENe. Small insertions and deletions were detected with NextGENe. Called SNVs were annotated with SeattleSeq Annotation and filtered with dbSNP130.

\section{Mutation analysis}

A mutation analysis of already-known genes for CFTD and LVNC and validation and segregation analyses of the selected variants obtained from exome sequencing, were performed by Sanger sequencing. 


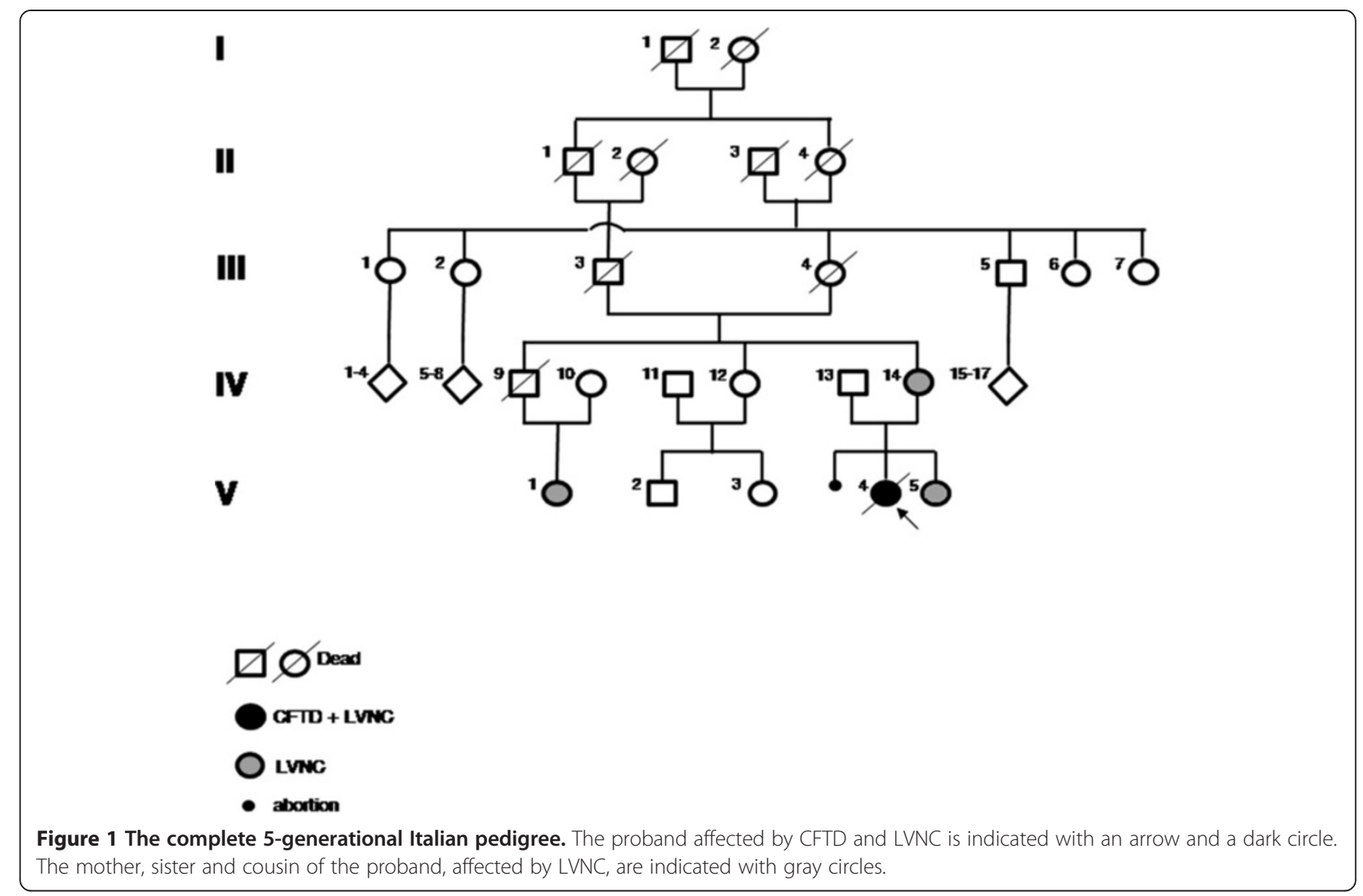

Each variant was amplified and sequenced, as described in our previous studies $[19,20]$. Variations were detected by multiple-sequence alignments using the Autoassembler program (Applied Biosystems).

The SIFT and PolyPhen2 software packages (http://sift.bii. a-star.edu.sg/www/SIFT_seq_submit2.html, http://genetics. bwh.harvard.edu/pph2/) were used to assess the deleterious effects of the mutations.

To assess the minor allele frequencies of the variants, the dbSNP (http://www.ncbi.nlm.nih.gov/snp) and the 1000 Genomes (http://www.ensembl.org/Homo_sapiens/) databases were surveyed.

\section{Expression analysis}

To examine the expression pattern of the $M Y H 7 B$ and ITGA7 genes, real-time PCR was performed on total RNAs from human adult tissues purchased from Stratagene and on total RNAs derived from the blood of family members using the LightCycler system DNA Engine Opticon 2 (MJ Research). The detailed protocol has already been published [21,22].

The expression levels were normalized to glyceraldehyde 3-phosphate dehydrogenase $(G A P D H)$ to account for differences in the starting material and in the cDNA reaction efficiency. Agarose gel electrophoresis was performed to further confirm the specific PCR products.
The $M Y H 7 B$ and ITGA7 transcript primers were the following:

MYH7B-cDNA-F 5' GTCTGGGTGCCTGATGAACA 3' MYH7B-cDNA-R 5' CTCGTTCAGGTGCGTCATCA 3' ITGA7-IS1-F 5' GGATGGTGGGGAATGGAAGT 3' ITGA7-IS1-R 5' GGTCAGCAGGGTCCAAAGTT 3' ITGA7-IS2-R 5' GCGGGGGTCCTGCTCTTCT 3' ITGA7-IS3-F 5' CAGAGGCAGGCAGAAGGATT 3'

The primer ITGA7-IS2-R was combined with either ITGA7-IS1-F or ITGA7-IS3-F.

The GAPDH primers were as follows: forward primer, 5' AGCCACATCGCTCAGACAC 3', and reverse primer, 5' GATCTCGCTCCTGGAAGATG 3'.

\section{Results}

\section{Clinical features of patient V-4}

The proband (V-4) is the first child of a family with a history of consanguinity (mother's parents are first-degree cousins) and diabetes in the maternal line (grandmother, one great-uncle, one uncle and a first-degree cousin) without neuromuscular diseases (Figure 1). Since birth, she manifested hypotonia, poor sucking and persistent crying. Persistent arterial duct and patent foramen ovale were diagnosed at birth but resolved spontaneously, as shown 
by echocardiography at the age of 1 month. At 3 months of age, she was diagnosed with congenital dislocation of the left hip, and at 17 months of age, she was hospitalized because of body weight below the third percentile and hypoglycemia. The failure to thrive was due to difficulties in chewing and swallowing. ECG showed a long QTc (478 msec). Echocardiography showed a slight left ventricular (LV) dilatation, a moderate reduction of the LV global function (ejection fraction $40 \%$ ), and a noncompacted aspect of the overall infero-lateral wall. She was administered ace-inhibitors, diuretics and digitalis, with a significant improvement in the clinical state. The karyotype and FISH were normal. The serum creatine kinase $(\mathrm{CK})$ was within the normal range. The patient was followed up for 8 years.

At 8 years of age, she was admitted at our department for a neuromuscular and cardiological evaluation.

The neurologic examination revealed the following: a waddling gait, an inability to stand up from a sitting position, a slight degree of weakness of the facial and masticator muscles, a mild weakness of the glutei and ileo-psoas and marked bilateral quadriceps. She developed marked scoliosis due to congenital hip dysplasia (Figure 2). Tendon reflexes were absent. Joints contractures had never been observed. A blood test confirmed hypoglycemia and

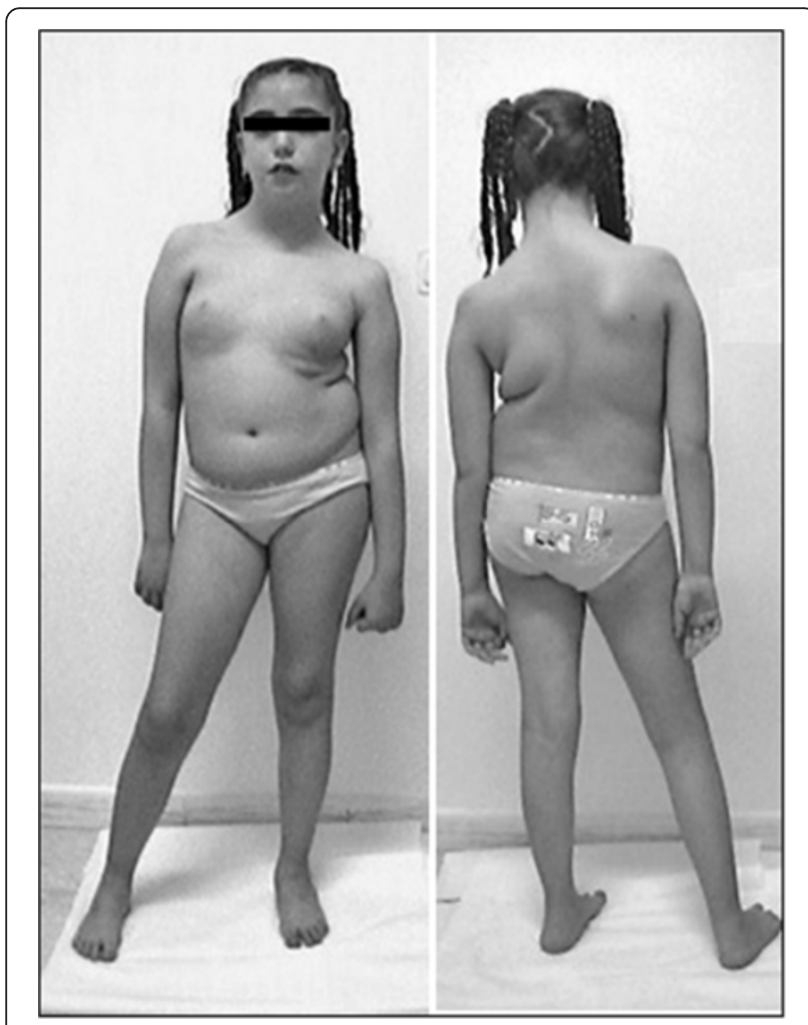

Figure $\mathbf{2}$ The proband at $\mathbf{1 0}$ years of age. Note the marked scoliosis, the leg length discrepancy and the left quadriceps hypotrophy; the abduction and elevation of the arms are impaired. normal levels of serum creatine kinase and isoenzymes. An EEG and a brain MRI showed no abnormality. A quadriceps muscle biopsy displayed a predominance of type 1 fibers (76\%), the mean diameter of which was $30 \%$ smaller than that of the type 2 fibers; the latter were hypertrophic for the age (mean diameter of $35 \mu \mathrm{m}$ ) (Figure 3). No internal nuclei, nemaline bodies or cores were observed. Dystrophin, emerin, calpain and lamin A/C were examined and found to be normal. This allowed us to exclude other neuromuscular disorders associated with the myopathy. These findings were compatible with the diagnosis of CFTD.

The cardiological investigation confirmed a long QTc and showed signs of left ventricular hypertrophy and repolarization abnormalities in the ECG. The echocardiography showed a dilated left ventricle (left ventricular end diastolic diameter $\mathrm{z}$-score +3 ) with hypertrabeculation of the antero-lateral, postero-lateral and inferior walls (non-compacted/compacted ratio $\sim 3$ ), a severe impairment of the LV global function (ejection fraction 35\%), a moderate mitral regurgitation, an abnormal mitral inflow pattern (E/A ratio 0.8), and a mild increase in the pulmonary artery pressure $(40 \mathrm{mmHg})$ with normal non-invasive pulmonary wedge pressure (E/Ea ratio 8 ) (Figures $4 \mathrm{~A}$ and 4B). A 24-hour Holter ECG monitoring revealed 5 short runs of supraventricular tachycardias. The patient was administered with carvedilol and ace-inhibitors, with the prescription of routine cardiac evaluations (every 4-6 months).

At the last cardiological evaluation (10 years of age), she was asymptomatic for dyspnoea (NYHA class I) and in therapy with carvedilol $6.25 \mathrm{mg} 1 / 2 \mathrm{cp} 2$ times/day, losartan $50 \mathrm{mg} 1 / 2 \mathrm{cp} /$ day, spironolattone $25 \mathrm{mg} 1 / 2 \mathrm{cp} /$ day, furosemide $25 \mathrm{mg} 1 \mathrm{cp} /$ day and cardioaspirin $100 \mathrm{mg}$ $1 \mathrm{cp} /$ day. The ECG confirmed a prolonged QTc (485 msec), and the echocardiography showed a non-compacted LV with $38 \%$ ejection fraction and slightly elevated pulmonary artery pressure $(45 \mathrm{mmHg})$. NT-proBNP was elevated (1858 pg/ml). A cardiac MRI analysis confirmed the diagnosis of LVNC, with a significant reduction of ejection fraction to $40 \%$ (Figure 4C).

One month before the cardiovascular appointment, the patient (11 years old) died suddenly early in the morning, while lying in bed.

\section{Gene identification strategy}

Whole-exome sequencing of the proband, affected by CFTD and LVNC, and her cousin, affected by LVNC, was used as a strategy to identify the responsible gene/s.

At first, we performed a thorough survey of all previously identified CFTD and LVNC genes to definitively exclude their involvement in the disease phenotype. Only intronic polymorphic variants were detected in TPM3, ACTA1, TPM2, MYL2, MYL3, G4.5, DTNA, ZASP, ACTC1, 


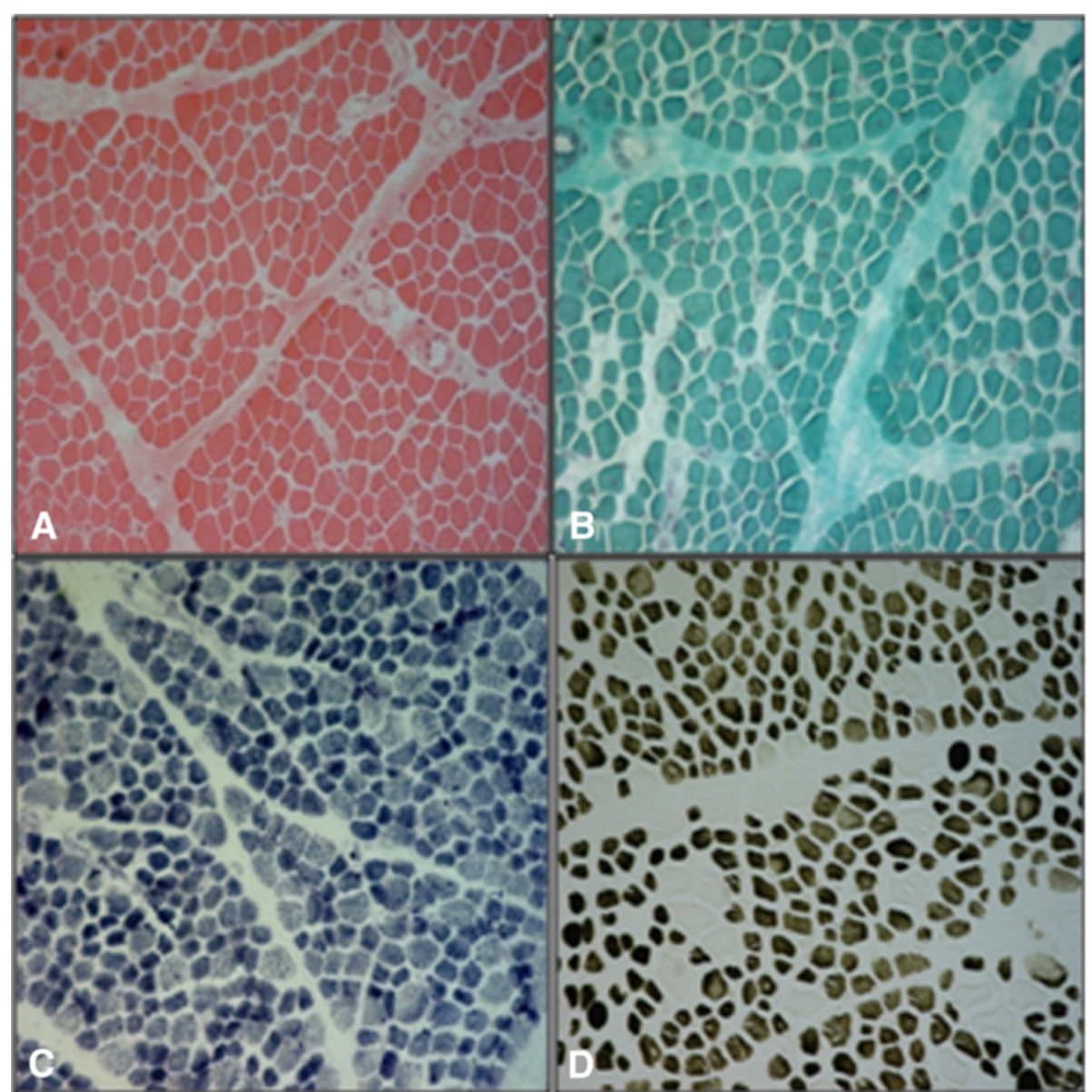

Figure 3 Right quadriceps muscle biopsy (6- $\mu$ m-thick cryostatic sections, 10x). (A) H-E: moderate fiber caliber variability and diffuse mild fibrosis; (B) Trichrome of Gomori: absence of mitochondrial accumulation; (C) NADH-Tr: no oxidative enzymes change; (D) ATPase pH 4.3: a clear predominance of type I (dark) fibers, which show diameters smaller than those of type 2 fibers (clear).

$M Y B P C 3$ and TMP1. Both intronic and coding polymorphic variants were detected in SEPN1, RYR1, MYH7, LMNA/C, TNNT2 and TNNI3 (Table 1). After filtering the data with dbSNP130 and data from six in-house exomes ( 1 healthy individual and 5 individuals with unrelated diseases; Table 2), we performed two models of analysis to follow both phenotypes: LVNC that segregated as dominant and CFTD that showed a recessive mode of inheritance. For the dominant model, we matched data from both individuals (V-1/V-4) to obtain shared variants. In total, 93 autosomal non-synonymous coding variants were shared between the two samples. Only 56 of these variants were confirmed by direct sequencing in the two patients. Ten variants showed complete segregation with the LVNC phenotype in the family but were also found in the healthy population with a minor allele frequency (MAF) $>1 \%$. Only one of these, R890C in myosin, heavy chain 7B, cardiac muscle, beta $(M Y H 7 B)$, was found in the 1000 Genomes database with a MAF $<0.05 \%$ and was predicted to be a deleterious change by bioinformatics tools.
For the recessive model, only the proband was considered to be affected. We then selected all genes carrying two mutations. In total, 46 homozygous changes were selected, but only 10 were confirmed by direct sequencing and analyzed in the family samples. It is important to note that the parents of individual IV-14 (mother of the proband) are first-degree cousins, so she is homozygous for many variants and represents a good sample to filter data derived from the proband. In fact, 9 of the 10 variants were excluded because they were homozygous both in the proband and in her mother, who did not show the CFTD phenotype. Only the E882K variant, in the integrin, alpha 7 (ITGA7) gene, segregated with the CFTD phenotype in the family.

The compound heterozygous model produced 50 genes, of which only 5 continued to carry two mutations after direct sequencing analysis; none of the 50 genes segregated with the disease phenotype in the family because they were also present in the sister of the proband (V5), who showed no CFTD phenotype. 


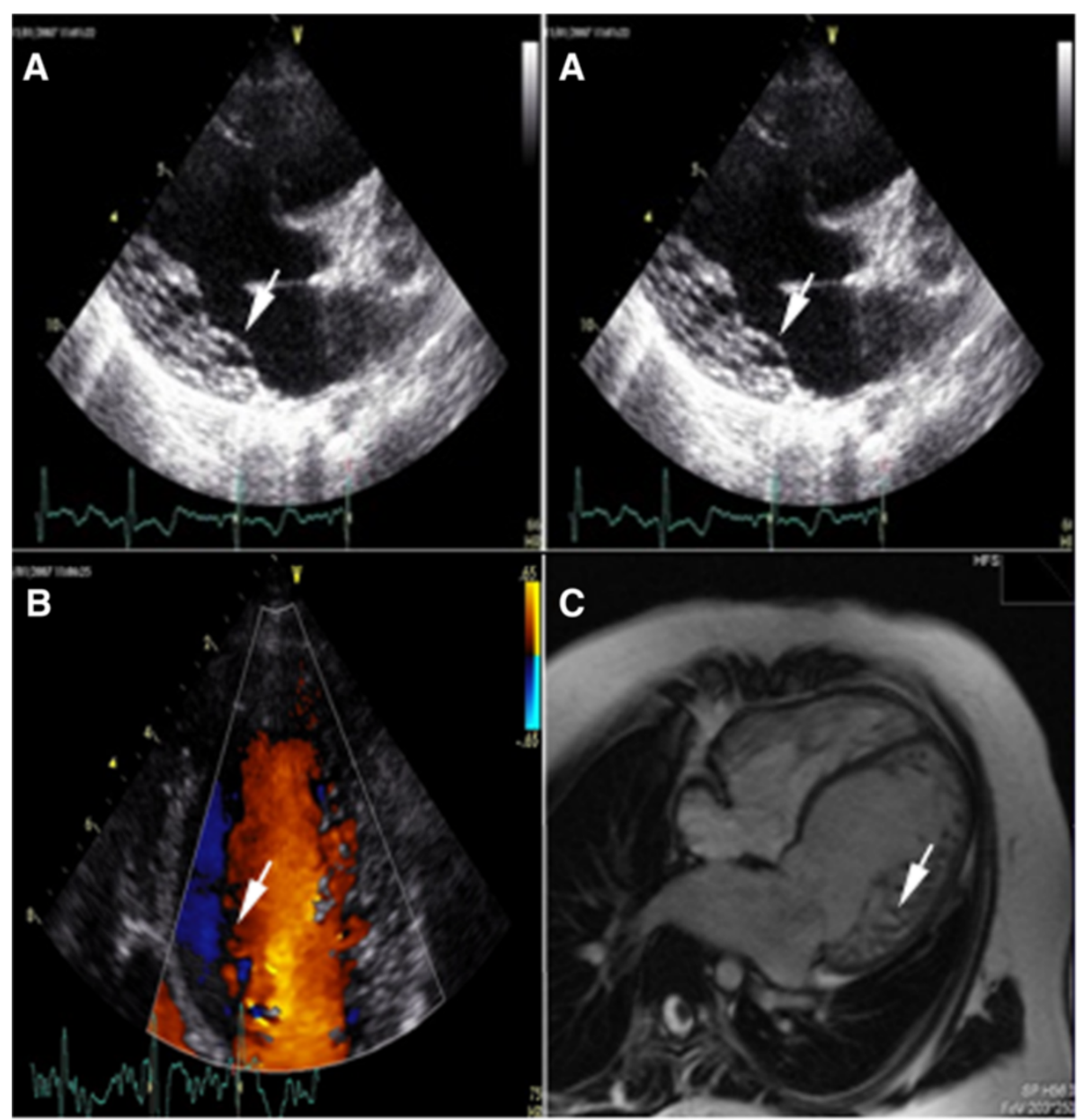

Figure 4 Cardiological features. (A) B-mode Echocardiography: ipertrabeculation/non-compaction left ventricle cardiomyopathy (white arrows). (B) Colordoppler-Echocardiography: blood flowing into the intertrabecular spaces (white arrow) communicating with the left ventricle lumen. (C) Cardiac MRI: a T1 weighted image clearly showing the left ventricle wall trabeculation (white arrow).

Table 1 Summary of the coding polymorphic variants found in CFTD-LVNC genes

\begin{tabular}{llll}
\hline Gene & Amino acid change & rs & MAF \\
\hline SEPN1 & C142Y & rs7349185 & $16 \%$ \\
SEPN1 & P391P & rs760597 & $20 \%$ \\
SEPN1 & N502K & rs2294228 & $28 \%$ \\
RYR1 & L198L & rs2229139 & $39 \%$ \\
RYR1 & P762P & rs3745847 & $38 \%$ \\
RYR1 & T981T & rs2228069 & $40 \%$ \\
RYR1 & N993N & rs2228070 & $24 \%$ \\
MYH7 & I989I & rs7157716 & $35 \%$ \\
MYH7 & K365K & rs735711 & $9 \%$ \\
MYH7 & F244F & rs2069542 & $22 \%$ \\
LMNA & H454H & rs4641 & $20 \%$ \\
TNNT2 & I101I & rs3729547 & $32 \%$ \\
TNN13 & E179E & rs3729841 & $4 \%$ \\
\hline$M A F m i n$ & &
\end{tabular}

MAF minor allele frequency.
The myosin heavy chain 7B (MYH7B) gene

MYH7B [GenBank: NG_016984.1] is located on chromosome 20 and is split into 45 exons, 43 of which code for protein. The nucleotide substitution c. $2668 \mathrm{C}>\mathrm{T}$ is located in exon 27 and causes the non-synonymous change of the amino acid arginine at position 890 into cysteine (R890C).

The inheritance of the R890C mutation was examined by Sanger sequencing of the DNA of the family individuals (Figure 5A). It was found to be homozygous in samples IV-14 and V-4 and to be heterozygous in IV-13, V-1 and $\mathrm{V}-5$; it was absent in IV-10. This finding suggests the segregation of the mutation with the LVNC disease phenotype in our family; however, its reduced penetrance and variable expressivity need to be considered because the mutation is also carried by the asymptomatic subject IV-13. To further validate our finding, we analyzed the R890C mutation in a panel of 24 unrelated LVNC patients from the same geographical area and found it in patient 
Table 2 Summary of the whole exome sequencing of CFTD-LVNC samples

\begin{tabular}{lll}
\hline Data & $\mathbf{V - 1}$ & $\mathbf{V - 4}$ \\
\hline Mapped Reads & $52,921,718(89 \%)$ & $42,081,251(90 \%)$ \\
SNV & 207.784 & 160,388 \\
dbSNPs & 140.798 & 110,677 \\
coding variants & 25.387 & 24,669 \\
Non synonym. (NS) & 13.480 & 13,089 \\
Frame-Shift & 196 & 182 \\
NS after filter dbSNPs & 3923 & 3784 \\
NS after filter 6 exomes* & 2983 & 2806 \\
Dominant Model & & \\
Dominant Model + validation** & & \\
Dominant Model (deleterious) & & 46 \\
Recessive Model homozygous & & 10 \\
Recessive Model + validation & & $\mathbf{1}$ \\
Recessive Model (deleterious) & & 50 \\
Recessive Model heterozygous & & 5 \\
Recessive Model + validation & & 0 \\
Recessive Model (deleterious) & & $\mathbf{1}$ \\
\hline SNV & &
\end{tabular}

SNV single nucleotide variation; *: non synonymous variants obtained after to filter with six in house genome; Dominant Model: number of gene in which at least one variant is shared between the two samples; Dominant Model + validation**: autosomal variants shared between the two samples and remaining after filtering with 1000 genome database and validated with direct sequencing; Dominant Model (deleterious): variants predicted as deleterious effect of the change with SIFT; Recessive Model: genes in which two alleles are mutated.

LVNC-14 (BG), who showed a classical LVNC phenotype with severe systolic dysfunction (25\%) (Figure 5A).

We then analyzed 600 chromosomes from an unrelated healthy population from the same geographical origin. This mutation was absent in this panel but was present in the 1000 Genomes database with a MAF $<0.006 \%$.

Most variants underlying rare Mendelian diseases either affect highly conserved sequences and/or are predicted to be deleterious. For this reason, we analyzed the R890C mutation with the SIFT and PolyPhen2 software to confirm a deleterious effect. At this position (R890), only arginine $(\mathrm{R})$, lysine $(\mathrm{K})$, serine $(\mathrm{S})$, asparagine $(\mathrm{N})$ or glutamine (Q) are tolerated (data not shown). The PolyPhen2 analysis supported the notion that this variant is "damaging", with the highest probability score of 1 .

The $M Y H 7 B$ gene belongs to the MYH gene family, which, in humans, also includes the MYH6 and MYH7 genes, both clustered on chromosome 14. Evolutionary conservation analysis shows high similarity for these proteins. Interestingly, the amino acid arginine $(\mathrm{R})$ at position 890 is highly conserved in all species, but in MYH6 and MYH7 and their homologs, the arginine (R) is replaced with lysine (K) (Figure 5B). However, lysine is one of the amino acids that SIFT predicted to be tolerated at this protein position. Using a bioinformatics simulation model (http://minnou.cchmc.org/), we demonstrated that the R890C mutation induces a conformational change in the MYH7B molecule [GenPept: AAI51243.1], leading to the alteration of the beta sheet and alpha helix structures (Figure 6).

A significant expression of the $M Y H 7 B$ gene was observed in heart and muscle tissues, and very low expression was observed in the brain, testes, ovary, liver and blood. No expression was observed in the kidney, lung and pancreas. No difference in expression was observed in blood RNA derived from both affected and healthy subjects from our family (Figure 5C).

\section{The integrin, alpha 7 (ITGA7) gene}

The ITGA7 gene [GeneBank: NG_012343.1] is located on chromosome 12 and is split into 28 exons, 26 of which code for protein. This gene has 23 transcripts, but only 10 are protein coding. The homozygous nucleotide substitution c. $2644 \mathrm{G}>\mathrm{A}$ is located in exon 20 and causes the non-synonymous change E882K in transcript 1 [GeneBank: NM_001144996.1]. The position of this mutation is E886K in isoform 2 [GeneBank: NM_002206.2] and E789K in isoform 3 [GeneBank: NM_001144997.1].

The inheritance of this mutation was examined by Sanger sequencing of the DNA of the family individuals. Our analysis confirmed that this mutation was present in a homozygous form only in the proband with the CFTD phenotype; her parents were heterozygous for this change (Figure 7A).

In the 1000 Genomes database, this change is reported as number rs144983062; the heterozygous genotype C/T 


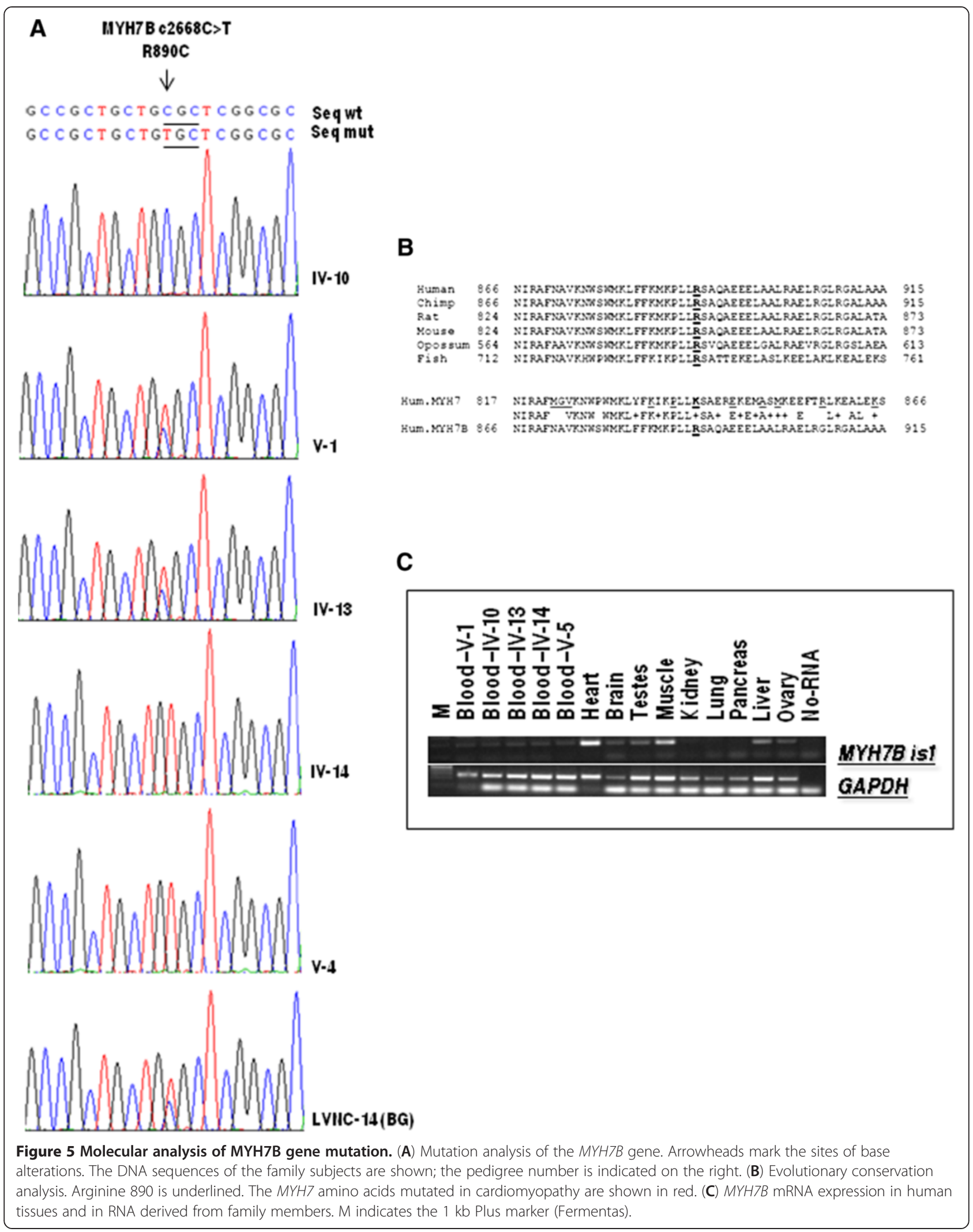




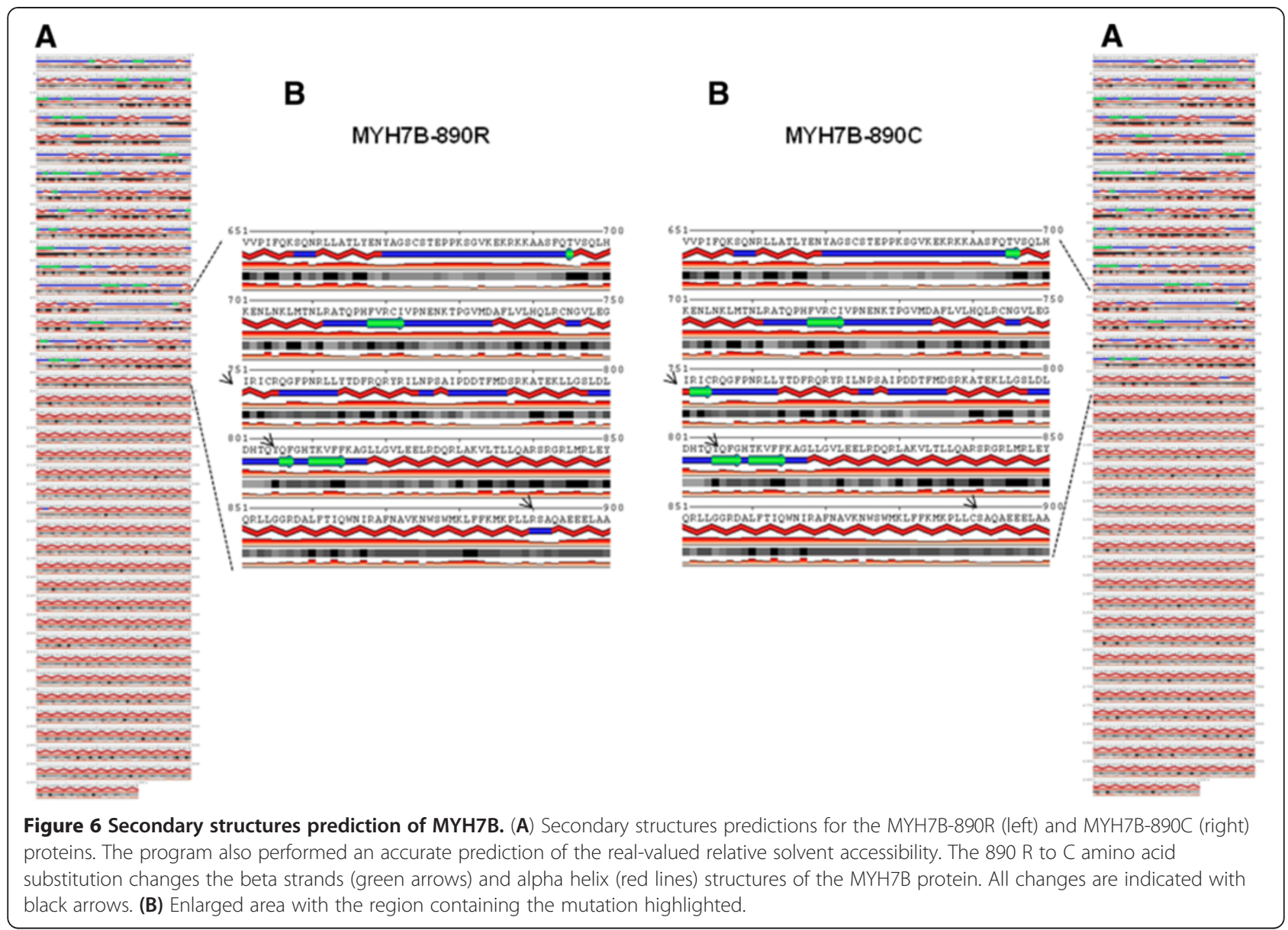

was found in 12 of the 4540 samples analyzed (freq of the CT genotype $=0.003$ ), and the homozygous genotype TT was not found. We analyzed 600 chromosomes from an unrelated healthy population from the same geographical area of the proband but did not find the mutation. Therefore, we analyzed the E882K mutation with the SIFT and PolyPhen2 software, which confirmed a deleterious effect of this variant. Evolutionary conservation analysis showed that the missense change E882K affects a highly conserved residue of the integrin alpha 7 protein (Figure 7B). Moreover, bioinformatics simulation modeling demonstrated that the E882K mutation induces a conformational change in the ITGA7 molecule, resulting in the alteration of the beta sheet structures (Figure 8).

Expression profiling was performed for three transcripts of the gene. Isoform 1 was significantly expressed only in heart, muscle, liver and blood tissues. No difference in expression was detected between blood RNAs derived from affected and healthy subjects of our family. Isoform 2 was significantly expressed in heart, muscle, testes and ovary tissues; low levels of expression were detected in the brain, kidney, lung, pancreas and liver; no expression was detected in the blood. Isoform 3 was detected at very low levels of expression only in the testes and kidney (Figure 7C).

\section{Discussion}

We describe an unusual association between CFTD and LVNC and link this unusual severe phenotype to digenic inheritance, a novel type of transmission that is emerging with the application of novel powerful genomic technologies, such as whole-exome sequencing [23]. The CFTD myopathy is a genetically heterogeneous disorder characterized by relative hypotrophy of type 1 muscle fibers compared to type 2 fibers in skeletal muscle biopsies [1]. The diagnosis of CFTD is made for exclusion because these findings are not specific and can be found in several neuromuscular diseases. The term "fiber size disproportion" has been suggested for describing this specific histological picture, reserving the term CFTD for those cases in which no secondary cause can be found [1]. Cardiovascular involvement has been rarely reported in patients with CFTD myopathy. Banwell et al. described 2 unrelated children with cardiac involvement [24]. One child, with a dilated type cardiomyopathy, developed an intractable congestive heart failure, necessitating cardiac 


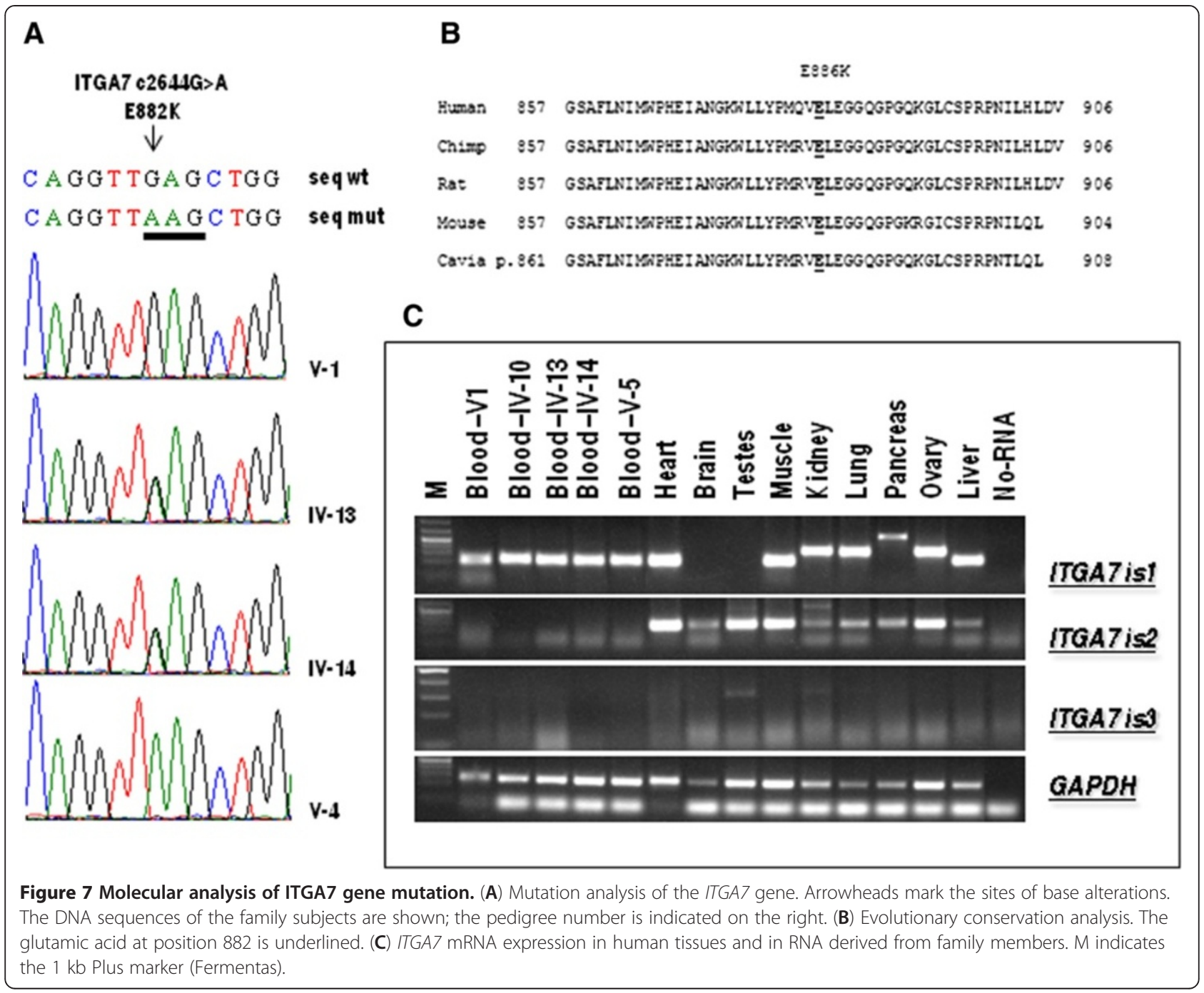

transplantation at the age of 13 years. The second, a 1 -year-old child without cardiomyopathy or congenital heart diseases, developed a high-rate atrial fibrillation, requiring treatment with digoxin. The association of DCM with CFTD has also been reported in two Japanese patients $[25,26]$. However, a defined geno-phenotype correlation has not been established in these cases. LVNC has been reported in patients with different types of NMDs, including dystrophinopathy, laminopathy, zaspopathy, myotonic dystrophy, Barth syndrome, Friedreich ataxia, CharcotMarie-Tooth disease, and metabolic and mitochondrial disorders $[27,28]$.

Nevertheless, considering the rarity of these pathological conditions, which are caused by a premature arrest of skeletal and cardiac muscle development, the hypothesis of a common pathogenesis is intriguing.

Our data suggest that rather than the action of a single gene, a synergic effect of homozygous mutations in two genes, i.e., myosin heavy chain 7B $(M Y H 7 B)$ and integrin alpha 7 (ITGA7), underlies the phenotype observed in the proband $(\mathrm{V}-4)$. Both genes are crucial for the physiological development of skeletal and cardiac muscles. However, a single-gene mutation responsible for both DCM/ LVNC and CFTD in other patients cannot be conclusively ruled out.

Myosin, the molecular motor responsible for muscle contraction, exists in multiple forms, which dictate muscle properties, such as shortening velocity and contractile force. The majority of MYH genes known to be present in mammals are associated in two highly conserved gene clusters [29,30]. MYH6 and MYH7 are two tandemly arrayed genes located on human chromosome 14, which code for the cardiac myosins, $\alpha$ - and $\beta-\mathrm{MYH} ; \beta-\mathrm{MYH}$ is also expressed in slow skeletal muscles. Another gene cluster, located on human chromosome 17, codes for the six skeletal myosins, including the adult fast 2A-, 2Xand 2B-MYH, the developmental embryonic and neonatal/ perinatal isoforms, and MYH13, an isoform expressed 


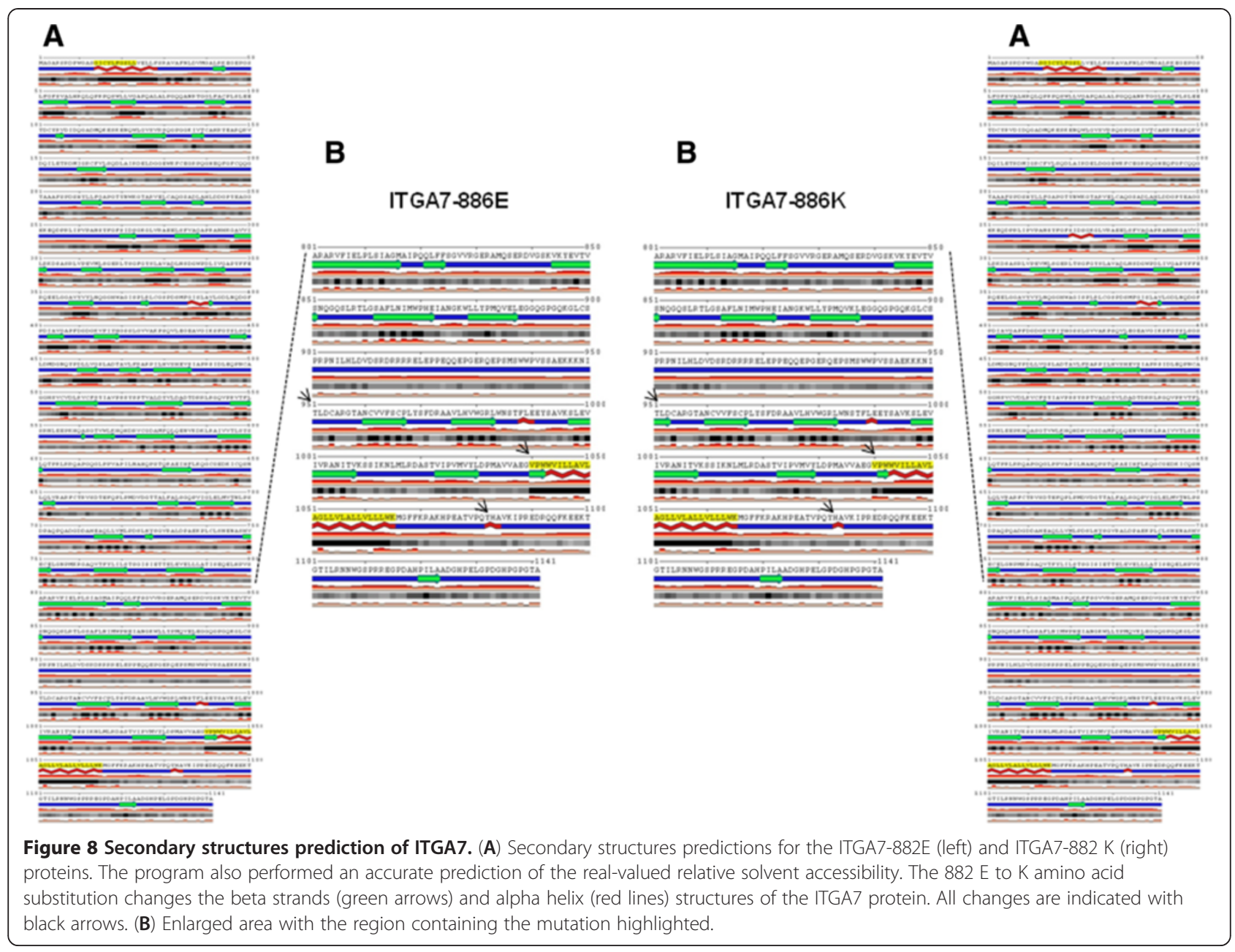

specifically in extraocular muscles. Three additional genes coding for sarcomeric MYHs, i.e., MYH7B, MYH15 and $M Y H 16$, have been discovered recently. We showed that $M Y H 7 B$ is expressed in the adult human heart and muscle; moreover, Warkman and coworkers recently determined that $M Y H 7 B$ is expressed in the myocardium [31]. Developmental analysis showed Myh7b expression in cardiac and skeletal muscles of Xenopus, chick and mouse embryos and in smooth muscle tissues during the later stages of mouse embryogenesis [31]. Heterozygous mutations in eight sarcomere proteins (MYH7, ACTC1, TNNT2, TNNI3, MYL2, MYL3, MYBPC3 and TPM1) have been identified in a significant proportion of patients with LVNC in adults and children [14-16,32,33]. Approximately $20 \%$ of LVNC patients carried a mutation in MYH7, which was the most prevalent LVNC disease gene; missense mutations were the most prevalent types of mutations in all sarcomere proteins analyzed [16,32]. LVNC is characterized by a trabecular meshwork and deep intertrabecular myocardial recesses communicating with the left ventricular (LV) cavity [18]. Clinical features range from a non-penetrant disease in adult carriers to heart failure, arrhythmia and thromboembolism [34,35]. The penetrance of a mutation is defined as the percentage of mutation carriers expressing a phenotype, and most autosomaldominant cardiomyopathies are characterized by incomplete penetrance or more age-related penetrance [36]. There is also variable expressivity in cardiomyopathies, and there can even be large differences among relatives of the same family (intrafamilial variability) who carry the same mutation. In our family, high phenotypic variability and reduced penetrance were observed.

This is the first indication that a mutation in the $M Y H 7 B$ gene causes LVNC cardiomyopathy. We demonstrated that the arginine at position 890 of the $M Y H 7 B$ gene is highly conserved in all species; this region is also conserved in the MYH7 gene, which, when mutated, causes LVNC. These data further support the concept that sarcomere genes are associated with LVNC.

Integrin $\alpha 7 \beta 1$ is a specific cellular receptor for the basement membrane protein laminin- 1 and for the laminin isoforms -2 and $-4[37,38]$. The $\alpha 7$ subunit is expressed 
mainly in skeletal and cardiac muscles and has been suggested to be involved in differentiation and migration processes during myogenesis [39-41].

Mice homozygous for a null allele of the Itga7 gene are viable and fertile, indicating that the $\alpha 7 \beta 1$ integrin is not essential for myogenesis. However, a histological analysis of skeletal muscle revealed typical symptoms of a progressive muscular dystrophy starting soon after birth, but with a distinct variability in different muscle types [42]. The knock-down of zebrafish Itga7 results in muscle fiber detachments similar to those observed in lama2 and lama4-deficient embryos [43]. The human deficiency in integrin $\alpha 7$ causes a mild disorder that is best characterized as congenital myopathy. Three patients with mutations in the ITGA7 gene have been described. One patient had splice mutations on both alleles: one mutation caused a 21-bp insertion in the conserved cysteine-rich region, and the other caused a 98-bp deletion. A second patient was a compound heterozygote for the same 98-bp deletion and had a 1-bp frame-shift deletion in the other allele. The third patient showed a marked deficiency in the ITGA7 mRNA, but no mutations in the coding region were described. In muscle biopsies, patients 1 and 3 showed a poorly defined congenital myopathy, which was associated with mental retardation in patient 1 . Patient 2 presented a clinical and pathological picture typical of muscular dystrophy, with substantial fatty acid replacement and fiber size variation (MIM 613204) [44].

Our proband (V-4) harbored a homozygous missense mutation in a highly conserved region of the protein. The typical pattern of CFTD was observed in the muscle biopsy, characterized by the predominance of type 1 fibers with smaller calibers than type 2 fibers, with no evidence of either congenital muscular dystrophy or muscular dystrophy. Clinically, she had no symptoms of a delay in mental development, which occurs only in a few cases of CFTD. Our proband and the above-mentioned cases, showing muscle disorders present from birth, all support the important role of ITGA7 in myogenesis. The differences in their phenotypes may be related to their diverse patterns of gene mutation.

\section{Conclusions}

This study identifies two novel disease genes. Mutation in $M Y H 7 B$ causes a classical LVNC phenotype, whereas mutation in ITGA7 causes CFTD. The synergic effect of these two mutations causes the severe phenotype observed in the proband. This study provides new insights into the genetics of cardiomyopathy and congenital myopathy.

\section{Competing interests}

All authors have non-financial interests that may be relevant to the submitted work.

\section{Authors' contributions}

$T E, D F, F N$ and FG performed the sequencing and expression analyses. SS, $G L, A V, D D, O F G P$, and $G D \mid$ recruited the family described herein and collected the clinical data. TE, SS, GL, FG and GDI oversaw all aspects of the research. TE and GDI initiated, planned and coordinated the study. TE, SS, GL and GDI wrote the manuscript. All authors read, edited and approved the final version of the manuscript.

\section{Acknowledgements}

We thank the participating patients and their families. We are grateful to G. Franzese and G. Panella for technical support.

\section{Author details}

"Institute of Genetics and Biophysics "Adriano Buzzati-Traverso", National Research Council of Italy, Naples, Italy. ${ }^{2}$ Department of Medical Sciences, Surgery, Neurological, Metabolic and Aging, Second University of Naples, Naples, Italy. ${ }^{3}$ Department of Cardiological Sciences, Second University of Naples, Naples, Italy. "Department of Neuro-sciences, "Santobono-Pausilipon" Hospital, Naples, Italy.

\section{Received: 5 April 2013 Accepted: 12 June 2013}

Published: 21 June 2013

\section{References}

1. Clarke NF, North KN: Congenital fiber type disproportion-30 years on. J Neuropathol Exp Neurol 2003, 62:977-989.

2. Laing NG, Clarke NF, Dye DE, Liyanage K, Walker KR, Kobayashi Y, Shimakawa S, Hagiwara T, Ouvrier R, Sparrow JC, Nishino I, North KN, Nonaka I: Actin mutations are one cause of congenital fibre type disproportion. Ann Neurol 2004, 56:689-694.

3. Clarke NF, Kidson W, Quijano-Roy S, Estournet B, Ferreiro A, Guicheney P, Manson Jl, Kornberg AJ, Shield LK, North KN: SEPN1: Associated with congenital fiber-type disproportion and insulin resistance. Ann Neurol 2006, 59:546-552.

4. Clarke NF, Ilkovski B, Cooper S, Valova VA, Robinson PJ, Nonaka I, Feng JJ, Marston S, North K: The pathogenesis of ACTA1-related congenital fiber type disproportion. Ann Neurol 2007, 61:552-561.

5. Clarke NF, Kolski H, Dye DE, Lim E, Smith RL, Patel R, Fahey MC, Bellance R, Romero NB, Johnson ES, Labarre-Vila A, Monnier N, Laing NG, North KN: Mutations in TPM3 are a common cause of congenital fiber type disproportion. Ann Neurol 2008, 63:329-337.

6. Brandis A, Aronica E, Goebel HH: TPM2 mutation. Neuromuscul Disord 2008, 18:1005.

7. Lawlor MW, Dechene ET, Roumm E, Geggel AS, Moghadaszadeh B, Beggs AH: Mutations of tropomyosin 3 (TPM3) are common and associated with type 1 myofiber hypotrophy in congenital fiber type disproportion. Hum Mutat 2009, 31:176-183.

8. Clarke NF, Waddell LB, Cooper ST, Perry M, Smith RL, Kornberg AJ, Muntoni F, Lillis S, Straub V, Bushby K, Guglieri M, King MD, Farrell MA, Marty I, Lunardi J, Monnier N, North KN: Recessive mutations in RYR1 are a common cause of congenital fiber type disproportion. Hum Mutat 2010, 31:E1544-E1550.

9. Towbin JA, Bowles NE: The failing heart. Nature 2002, 415:227-233.

10. Chen R, Tsuji T, Ichida F, Bowles KR, Yu X, Watanabe S, Hirono K, Tsubata S, Hamamichi Y, Ohta J, Imai Y, Bowles NE, Miyawaki T, Towbin JA, Noncompaction study collaborators: Mutation analysis of the G4.5 gene in patients with isolated left ventricular noncompaction. Mol Genet Metab 2002, 77:319-325.

11. Ichida F, Tsubata S, Bowles KR, Haneda N, Uese K, Miyawaki T, Dreyer WJ, Messina J, Li H, Bowles NE, Towbin JA: Novel gene mutations in patients with left ventricular noncompaction or Barth syndrome. Circulation 2001 103:1256-1263.

12. Vatta M, Mohapatra B, Jimenez S, Sanchez X, Faulkner G, Perles Z, Sinagra G, Lin JH, Vu TM, Zhou Q, Bowles KR, Di Lenarda A, Schimmenti L, Fox M, Chrisco MA, Murphy RT, McKenna W, Elliott P, Bowles NE, Chen J, Valle G, Towbin JA: Mutations in Cypher/ZASP in patients with dilated cardiomyopathy and left ventricular noncompaction. J Am Coll Cardiol 2003, 42:2014-2027.

13. Hermida-Prieto M, Monserrat L, Castro-Beiras A, Laredo R, Soler R, Peteiro J, Rodríguez E, Bouzas B, Alvarez N, Muñiz J, Crespo-Leiro M: Familial dilated cardiomyopathy and isolated left ventricular noncompaction associated with lamin A/C gene mutations. Am J Cardiol 2004, 94:50-54. 
14. Budde BS, Binner P, Waldmüller S, Höhne W, Blankenfeldt W, Hassfeld S, Brömsen J, Dermintzoglou A, Wieczorek M, May E, Kirst E, Selignow C, Rackebrandt K, Müller M, Goody RS, Vosberg HP, Nürnberg P, Scheffold T: Noncompaction of the ventricular myocardium is associated with a de novo mutation in the beta-myosin heavy chain gene. PLoS One 2007, 2(12):e1362.

15. Klaassen S, Probst S, Oechslin E, Gerull B, Krings G, Schuler P, Greutmann M, Hürlimann D, Yegitbasi M, Pons L, Gramlich M, Drenckhahn JD, Heuser A Berger $F$, Jenni $R$, Thierfelder $L$ : Mutations in sarcomere protein genes in left ventricular noncompaction. Circulation 2008, 117(22):2893-2901.

16. Probst $S$, Oechslin E, Schuler P, Greutmann M, Boyé P, Knirsch W, Berger F, Thierfelder $L$, Jenni $R$, Klaassen $S$ : Sarcomere gene mutations in isolated left ventricular noncompaction cardiomyopathy do not predict clinical phenotype. Circ Cardiovasc Genet 2011, 4(4):367-374.

17. Ng SB, Buckingham KJ, Lee C, Bigham AW, Tabor HK, Dent KM, Huff CD, Shannon PT, Jabs EW, Nickerson DA, Shendure J, Bamshad MJ: Exome sequencing identifies the cause of a mendelian disorder. Nat Genet 2010, 42:30-36.

18. Jenni R, Oechslin E, Schneider J, Attenhofer Jost C, Kaufmann PA: Echocardiographic and pathoanatomical characteristics of isolated left ventricular non-compaction: a step towards classification as a distinct cardiomyopathy. Heart 2001, 86(6):666-671.

19. Gennari L, Gianfrancesco F, Di Stefano M, Rendina D, Merlotti D, Esposito T, Gallone S, Fusco P, Rainero I, Fenoglio P, Mancini M, Martini G, Bergui S, De Filippo G, Isaia G, Strazzullo P, Nuti R, Mossetti G: SQSTM1 gene analysis and gene-environment interaction in Paget's disease of bone. J Bone Miner Res 2010, 25:1375-1384

20. Gianfrancesco F, Rendina D, Di Stefano M, Mingione A, Esposito T, Merlotti D, Gallone S, Magliocca S, Goode A, Formicola D, Morello G, Layfield R, Frattini A, De Filippo G, Nuti R, Searle M, Strazzullo P, Isaia G, Mossetti G, Gennari L: A non synonymous TNFRSF11A variation increases NFKB activity and the severity of Paget's disease. J Bone Miner Res 2012, 27(2):443-452.

21. Esposito T, Magliocca S, Formicola D, Gianfrancesco F: piR_015520 belongs to Piwi-associated RNAs regulates expression of the human melatonin receptor 1A gene. PLoS One 2011, 6:e22727

22. Esposito T, Rendina D, Aloia A, Formicola D, Magliocca S, De Filippo G, Muscariello R, Mossetti G, Gianfrancesco F, Strazzullo P: The melatonin receptor 1A (MTNR1A) gene is associated with recurrent and idiopathic calcium nephrolithiasis. Nephrol Dial Transplant 2012, 27(1):210-218.

23. Lupski JR: Digenic inheritance and Mendelian disease. Nat Genet 2012, 44(12):1291-1292.

24. Banwell BL, Becker LE, Jay V, Taylor GP, Vajsar J: Cardiac manifestations of congenital fiber-type disproportion myopathy. J Child Neurol 1999, 14:83-87.

25. Fujita K, Nakano S, Yamamoto H, Ito H, Ito H, Goto Y, Kusaka H: An adult case of congenital fiber type disproportion (CFTD) with cardiomyopathy. Rinsho Shinkeigaku 2005, 45(5):380-382.

26. Okamoto N, Toribe $Y$, Nakajima T, Okinaga T, Kurosawa K, Nonaka I, Shimokawa O, Matsumoto N: A girl with 1 p36 deletion syndrome and congenital fiber type disproportion myopathy. J Hum Genet 2002, 47:556-559.

27. Stollberger C, Finsterer J: Left ventricular hypertrabeculation /noncompaction. J Am Soc Echocardiogr 2004, 17(1):91-100.

28. Finsterer J: Cardiogenetics, neurogenetics, and pathogenetics of left ventricular hypertrabeculation/noncompaction. Pediatr Cardiol 2009, 30:659-681

29. Weiss A, Schiaffino S, Leinwand LA: Comparative sequence analysis of the complete human sarcomeric myosin heavy chain family: implications for functional diversity. J Mol Biol 1999, 290(1):61-75

30. Weiss A, McDonough D, Wertman B, Acakpo-Satchivi L, Montgomery K, Kucherlapati $R$, Leinwand $L$, Krauter $K$ : Organization of human and mouse skeletal myosin heavy chain gene clusters is highly conserved. Proc Natl Acad Sci USA 1999, 96(6):2958-2963.

31. Warkman AS, Whitman SA, Miller MK, Garriock RJ, Schwach CM, Gregorio CC, Krieg PA: Developmental expression and cardiac transcriptional regulation of Myh7b, a third myosin heavy chain in the vertebrate heart. Cytoskeleton 2012, 69(5):324-335.

32. Hoedemaekers YM, Caliskan K, Michels M, Frohn-Mulder I, van der Smagt JJ, Phefferkorn JE, Wessels MW, ten Cate FJ, Sijbrands EJ, Dooijes D, MajoorKrakauer DF: The importance of genetic counseling, DNA diagnostics, and cardiologic family screening in left ventricular noncompaction cardiomyopathy. Circ Cardiovasc Genet 2010, 3(3):232-239.
33. Walsh R, Rutland C, Thomas R, Loughna S: Cardiomyopathy: a systematic review of disease-causing mutations in myosin heavy chain 7 and their phenotypic manifestations. Cardiology 2010, 115(1):49-60.

34. Johnson MT, Zhang S, Gilkeson R, Ameduri R, Siwik E, Patel CR, Chebotarev $\mathrm{O}$, Kenton AB, Bowles KR, Towbin JA, Robin NH, Brozovich F, Hoit BD: Intrafamilial variability of noncompaction of the ventricular myocardium. Am Heart J 2006, 151(5):1012.e7-14

35. Jenni $\mathrm{R}$, Oechslin EN, van der Loo B: Isolated ventricular non-compaction of the myocardium in adults. Heart 2007, 93(1):11-15.

36. Charron P, Arad M, Arbustini E, Basso C, Bilinska Z, Elliott P, Helio T, Keren A, McKenna WJ, Monserrat L, Pankuweit S, Perrot A, Rapezzi C, Ristic A Seggewiss H, van Langen I, Tavazzi L, European Society of Cardiology Working Group on Myocardial and Pericardial Diseases: Genetic counselling and testing in cardiomyopathies: a position statement of the European Society of Cardiology Working Group on Myocardial and Pericardial Diseases. Eur Heart J 2010, 31(22):2715-2726.

37. Kramer RH, Vu MP, Cheng YF, Ramos DM, Timpl R, Waleh N: Laminin-binding integrin alpha 7 beta 1: functional characterization and expression in normal and malignant melanocytes. Cell Regul 1991, 2(10):805-817.

38. Yao CC, Ziober BL, Squillace RM, Kramer RH: Alpha7 integrin mediates cell adhesion and migration on specific laminin isoforms. J Biol Chem 1996, 271(41):25598-25603.

39. Song WK, Wang W, Foster RF, Bielser DA, Kaufman SJ: H36-alpha 7 is a novel integrin alpha chain that is developmentally regulated during skeletal myogenesis. J Cell Biol 1992, 117(3):643-657.

40. Ocalan M, Goodman SL, Kühl U, Hauschka SD, von der Mark K: Laminin alters cell shape and stimulates motility and proliferation of murine skeletal myoblasts. Dev Biol 1988, 125(1):158-167.

41. Goodman SL, Risse G, von der Mark K: The E8 subfragment of laminin promotes locomotion of myoblasts over extracellular matrix. J Cell Biol 1989, 109(2):799-809.

42. Mayer U, Saher G, Fässler R, Bornemann A, Echtermeyer F, von der Mark H, Miosge N, Pöschl E, von der Mark K: Absence of integrin alpha 7 causes a novel form of muscular dystrophy. Nat Genet 1997, 17(3):318-323.

43. Postel R, Vakeel P, Topczewski J, Knöll R, Bakkers J: Zebrafish integrin-linked kinase is required in skeletal muscles for strengthening the integrin-ECM adhesion complex. Dev Biol 2008, 318(1):92-101.

44. Hayashi YK, Chou FL, Engvall E, Ogawa M, Matsuda C, Hirabayashi S, Yokochi K, Ziober BL, Kramer RH, Kaufman SJ, Ozawa E, Goto Y, Nonaka I, Tsukahara T, Wang JZ, Hoffman EP, Arahata K: Mutations in the integrin alpha7 gene cause congenital myopathy. Nat Genet 1998, 19(1):94-97.

doi:10.1186/1750-1172-8-91

Cite this article as: Esposito et al.: Digenic mutational inheritance of the integrin alpha 7 and the myosin heavy chain 7B genes causes congenital myopathy with left ventricular non-compact cardiomyopathy. Orphanet Journal of Rare Diseases 2013 8:91.

\section{Submit your next manuscript to BioMed Central and take full advantage of:}

- Convenient online submission

- Thorough peer review

- No space constraints or color figure charges

- Immediate publication on acceptance

- Inclusion in PubMed, CAS, Scopus and Google Scholar

- Research which is freely available for redistribution 